\title{
BRAIN MR IMAGE SEGMENTATION BY MODIFIED ACTIVE CONTOURS AND CONTOURLET TRANSFORM
}

\author{
P. Nageswara Reddy ${ }^{1}$, C.P.V.N.J. Mohan Rao $^{2}$ and Ch. Satyanarayana ${ }^{3}$ \\ ${ }^{1,3}$ Jawaharlal Nehru Technological University College of Engineering, India \\ ${ }^{2}$ Department of Computer Science and Engineering, Avanthi Institute of Engineering and Technology, India
}

\begin{abstract}
Multiresolution analysis is often used for image representation and processing because it can represent image at the split resolution and scale space. In this paper, a novel medical image segmentation algorithm is proposed that combines contourlet transform and modified active contour model. This method has a new energy formulation by representing the image with the coefficients of a contourlet transform. This results fast and accurate convergence of the contour towards the object boundary. Medical image segmentation using contourlet transforms has shown significant improvement towards the weak and blurred edges of the Magnetic Resonance Image (MRI). Also, the computational complexity is less compared to using traditional level sets and variational level sets for medical image segmentation. The special property of the contourlet transform is that, the directional information is preserved in each sub-band and is captured by computing its energy. This energy is capable of enhancing weak and complex boundaries in details. Performance of medical image segmentation algorithm using contourlet transform is compared with other deformable models in terms of various performance measures.
\end{abstract}

Keywords:

Multiresolution, Contourlet Transform, MRI, Active Contours, Segmentation

\section{INTRODUCTION}

Image segmentation is a fundamental but important task in many image processing and computer vision applications, because the segmentation result will directly affect the performance of the subsequent processing steps. Image segmentation occurs as a fundamental early vision processing task in many important medical applications. A significant challenge of medical image segmentation is intensity inhomogeneity due to the bias field, which is caused by various factors, such as the spatial variations in illumination and the imperfection of imaging devices.

A large number of techniques and algorithms have been proposed in the literature [1]-[3]. Medical image segmentation is the process of labeling each pixel or voxel in a medical image dataset to indicate its tissue type or anatomical structure. The labels that result from this process have a wide variety of applications in medical research and visualization. Segmentation of an anatomical structure from medical image amounts to identifying a region or boundary of an image corresponding to the desired structure. There is an immense array of scientific literature focusing on the task of medical image segmentation [4]-[6] and it has received significant attention, due to many practical applications of segmentation results. By making use of medical image segmentation approaches accurate, repeatable, quantitative data must be efficiently extracted in order to support the spectrum of biomedical investigations and clinical activities, from diagnosis, to radiotherapy to surgery. Many different image segmentation algorithms have been developed in the past several decades but it remains a complex and challenging task.

Medical image segmentation models can be used to recognize pathologies, such as tumors, neuro-anatomical structures etc. Identifying the location is an important aspect for the doctors because the evolution of the disease, its cause, and the available treatment options are mainly depending on the diseased anatomical structure. A segmentation technique depends on the specific applications, imaging methods and other factors pertaining to diagnosis. At present no single segmentation method that yields acceptable results for every medical image. The need of segmentation is to provide richer information than that which exists in the original medical images alone. Segmentations are also valuable in building anatomical atlases, analyzing shapes of anatomical structures, and tracking anatomical changes over time. A fully segmented scan allows surgeons to both better qualitatively visualize the shapes and relative positions of internal structures and more accurately measure their volumes and distances quantitatively.

In this paper, we propose a technique combining improved variational level set and contourlet transform. We define energy functional with a local intensity fitting term, which is important for attracting the contour towards object boundaries, which incorporates global image information to improve the robustness of the proposed model. This energy can also be incorporated into a level set regularization term that is necessary for correct evaluation in the corresponding level set model. This technique has a new energy framework by indicating the image with the distribution coefficients of a multiresolution coefficients called contourlet transform. In the associated contour evolution, the speed of the contour is driven by a local intensity fitting energy and a global intensity force, produced by the local and global terms in the proposed energy model respectively. When the proximity of the contour is very close to object boundaries, the local intensity fitting force becomes strong, which attracts the contour towards the boundary and finally trap the contour at object boundaries. This energy plays a key role in accurately locating object boundaries. The global intensity force is very strong when the contour is far away from object boundaries, and it allows more flexible initialization of contours by using global image information.

In this paper, various techniques of medical image segmentation, their approaches and limitations are briefly discussed in section 2. Materials and methods as part of reprocessing the image and variational level set for segmentation were explained in section 3. Proposed method combining contourlet transform and level set were provided in section 4 . 
Results and discussion of the proposed method was discussed in section 5. Concluding remarks are given in section 6.

\section{RELATED WORK}

There have been multitudes of approaches proposed for automatic object segmentation from biomedical images [7]-[9]. However, automatic segmentation remains to be the challenging task. Some of the main challenges include the extraction of object boundaries or regions from images with noise and intensity inhomogeneity, which often exist in biomedical images due to factors such as sampling artifacts. In active contours models, contour attraction towards the weak and blurred edges can be easily achieved without any re-initialization. Also, the computational complexity is less compared to traditional level sets and variational level sets [10]-[13]. This particular class of multi-scale segmentation technique based on wavelets relies on multi-scale representations of images. Such methods are typically based on image transformations that change image resolution, being able to segment objects of different sizes. In general small details are detected in higher resolution images, while larger objects are segmented in courser images. The advantages of using wavelets are that, it allows an approximation of the tumor boundaries by increasing or decreasing the number of wavelets coefficients. Further, the wavelet decomposition is a complete representation, since it allows a perfect reconstruction of the original image [14], [15]. However, wavelets are good at isolating the discontinuities at edge points of an image, but cannot see the smoothness along the contours. Further, wavelets can capture only limited directional information like horizontal, vertical and diagonal. So these limitations restrict using wavelets for specific applications.

To overcome these limitations, multi-scale and directional representations, which can capture the intrinsic geometrical structures such as smooth contours in medical images, have been developed [16]-[18]. The main drawback in these methods is that there is no flexibility in selection of different number and directions at each scale. To overcome this deficiency, Do and Vetterli [19] proposed the Contourlet Transform (CT) based on 2D multi-scale and directional filter bank that can deal efficiently with images having smooth contours which are common in medical images. The special property of the contourlet transform is that, the directional information is preserved in each sub-band and is captured by computing its energy. This energy is capable of enhancing weak and complex boundaries in details. Further, due its directional image expansion property, smoothness along the contours can be easily achieved. The contourlet transform has better performance in enhancing weak and complex edges in medical images than wavelets and other multiresolution transforms. Hybrid models (combining watershed and deformable models) are computationally efficient but they are poor in convergence, due to the lack of details along the edges of the object boundaries. However, hybrid model combining contourlet with variational level sets is superior in terms of convergence.

Huang and Zeng [20] proposed the modified method of an active contour model for the Segmentation of images with intensity inhomogeneities and bias field estimation to obtain accurate segmentation results and an accurate estimation of the bias field. The energy function is incorporated into a level set formulation with a level set regularization term, and the energy minimization is conducted by a level set evolution process. Further, a novel region-based active contour model in the level set formulation for medical image segmentation [21], [22] to define a unified fitting energy frame work based on Gaussian probability distributions to obtain the maximum a posteriori probability (MAP) estimation.

\section{MATERIALS AND METHODS}

\subsection{PREPROCESSING}

The Contourlet transform is one of the new geometrical image transforms, which represents images containing contours and textures. The contourlet transform effectively captures smooth contours images that are the dominant feature in natural images. The main difference between contourlets and other multi scale directional systems is that the contourlet transform allows for different and flexible number of directions at each scale, while achieving nearly critical sampling. Candes and Donoho [16] showed that wavelets perform well for objects with point singularities in 1D and 2D. Orthogonal wavelets capture only horizontally, vertically, and diagonally directed discontinuities. These orientations may not preserve enough directional information in medical images. Ridgelets analysis, on the other hand, is an appropriate transform to catch radial directional details in frequency domain. Ridgelets are very effective in detecting linear radial structures, but those structures are not dominant in medical images. An extension of Ridgelets transform introduced by Candes and Donoho [16] is the curvelet transform. Curvelet transform overcomes the directionality lack of 2-D wavelets by geometrically representing smoothness of contours. But, theoretically there are no significant differences in the time performance in compared with wavelets transform. Also, curvelets are highly anisotropic at fine scales, but curvelet construction is simple in continuous domain and complex in discrete domain. Further, they are slow as compared to contourlet transforms.

Do and Vetterli [19] constructed a discrete domain multiresolution and multidirectional expansion using nonseparable filter banks to obtain sparse expansions for typical images having smooth contours. In this double filter bank, the Laplacian pyramid (LP) is first used to capture the point discontinuities, followed by a Directional Filter Bank (DFB) to link point discontinuities into linear structures. The overall result is an image expansion using basic elements like contour segments, named contourlets. In particular, contourlets have elongated supports at various scales, directions and aspect ratios. Making use of these efficient properties of contourlet transform, a new segmentation algorithm is proposed. In this algorithm contourlets represents features of medical images efficiently compared to other transforms. The Fig. 1 shows a block diagram of the contourlet transform. It consists of two steps: Sub-bands decomposition and directional transform. A Laplacian Pyramid (LP) is first used to capture point discontinuities, then followed by a Directional Filter Bank (DFB) to link point discontinuity into linear structure.

First decomposition is the LP stage and second stage is DFB decomposition. The LP decomposition at each stage gives a sampled low pass type of the original and the difference between 
the original and the estimated, results a band pass image. Assume $f(x, y)$ represents the original image and its sampled version of low pass filtered image is $f_{\text {low }}(x, y)$.

The estimation error results as a band pass image $L_{l o w}(x, y)$ and the estimated is as follows;

$$
\overline{f_{l o}}=f(x, y)-f^{\prime}(x, y)
$$

where, $f^{\prime}(x, y)$ indicates the estimation of $f(x, y)$.

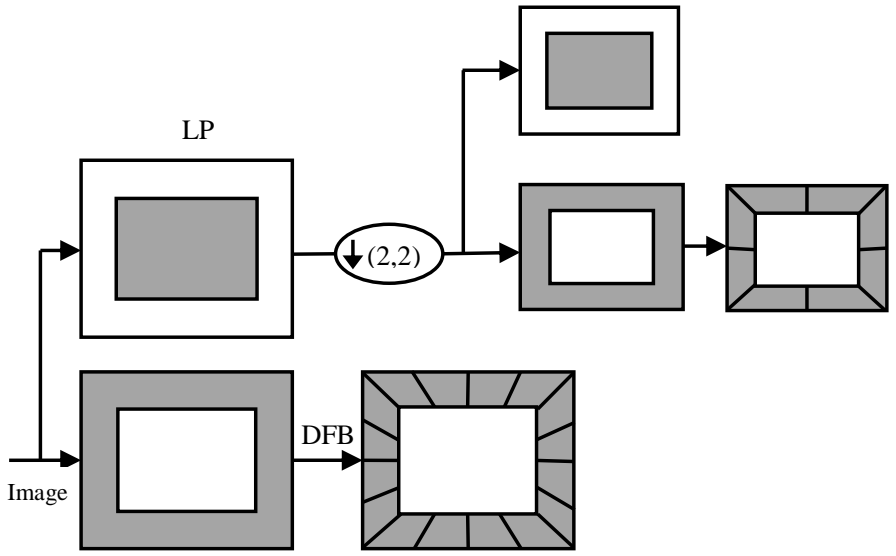

Fig.1. Block diagram of the contourlet transform. The image is first decomposed into sub bands through Laplacian Pyramid and then each band pass or detail image is analyzed by the directional filter banks [19]

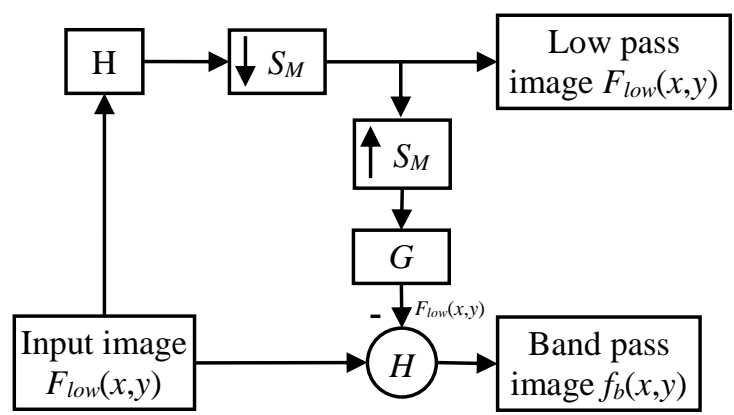

(a) One level LP decomposition

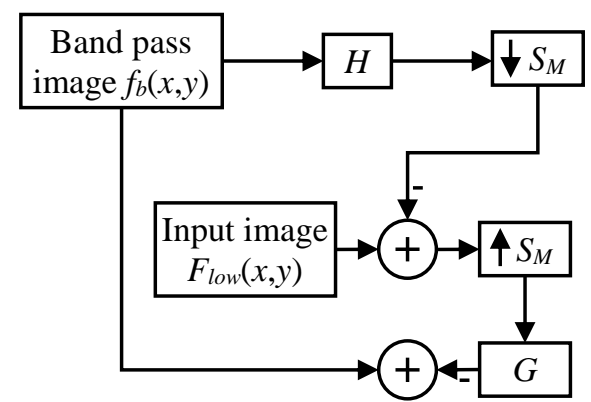

(b) One level LP reconstruction [23]

Fig.2. One level low pass (LP) decomposition and reconstruction

Encoding step is performed on $\bar{f}_{l o}$ as it is largely decorrelated. Further encoding can be carried out by applying Eq.(1) on $L_{\text {low }}(x, y)$ iteratively to get $n$ levels and $n$ represents the pyramidal level number. In Fig.2, $H$ and $G$ represents analysis and synthesis low pass filters respectively. The $S_{M}$ represents the sampling matrix. One level low pass (LP) decomposition and reconstruction are shown in Fig.2(a) and Fig.2(b) respectively.

The DFB is efficiently implemented via $n$ level tree-structured decomposition that leads to $2^{n}$ sub-bands with $2^{3}=8$ wedgeshaped frequency partition as shown in Fig.3.

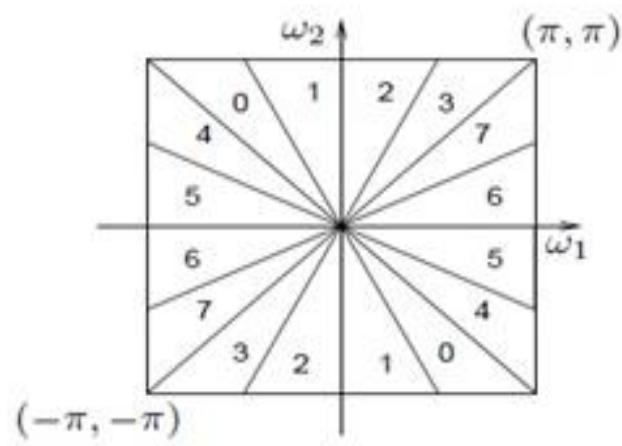

Fig.3. Directional filter bank frequencies partitioning [19]

\subsection{VARIATIONAL LEVEL SET MODEL}

The energy minimization function in the following Equation generates a segmentation method, which attracts the contour towards the boundaries of the image object. The energy function is defined in the form containing image energy and shape energy as,

$$
E(\phi)=E_{\text {image }}(\phi)+v E_{\text {shape }}(\phi)
$$

where, $E_{\text {image }}(\phi)$ is the image based term, $E_{\text {shape }}(\phi)$ is the shape, and $v$ is a constant that regulates the of the shape based energy.

The energy functional minimization $E(\phi)$ can be called as a segmentation model that simultaneously maximizes the accuracy of the object boundaries indicated by the evolving shape.

Therefore the new image based energy functional can be shown as;

$$
E_{\text {image }}(\phi)=+\alpha \int_{\Omega} g(x)|\nabla H(\phi)| d x+\int_{\Omega} G(x) H(\phi) d x
$$

where, $\alpha$ regulates controls the smoothness of the contours, $H$ is the Heaviside function and $G(x)$ represents the gradient vector interaction field.

The shape energy functional based on the shape distance measures between the evolving shape and training shapes $\phi_{i}$ is as follows;

$$
E_{\text {shape }}(\phi)=\int_{\Omega}\left(H\left(\phi\left(x+\mu_{\phi}\right)\right)-h\left(\phi_{i}\right)\right)^{2} d x
$$

where, $\mu_{\phi}$ denotes the centre of gravity of the shape $\phi$ and can be defined as:

$$
\mu_{\phi}=\int x h(\phi) d x
$$

The energy function in Eq.(2) generates a segmentation model, which attracts the active contour towards object boundaries. Therefore energy function of the Eq.(2) can be written as,

$$
E=\int f(\phi), \nabla(\phi) d x
$$

Then Euler-Lagrange equation is defined as, 


$$
\begin{gathered}
\frac{\partial f}{\partial \phi}-\nabla \cdot \frac{\partial f}{\partial \nabla \phi}=0 \\
\frac{\partial f}{\partial \phi}-\frac{\partial E_{\text {image }}(\phi)}{\partial \phi}-v \cdot \frac{\partial E_{\text {shape }}(\phi)}{\partial \phi}
\end{gathered}
$$

The image based gradient flow is as,

$$
\frac{\partial E_{\text {image }}(\phi)}{\partial \phi}=-\alpha \nabla\left(g(x) \frac{\nabla \phi(x)}{\phi(x)}\right) \delta(\phi(x))+G(x) \cdot \delta(\phi(x))
$$

where, $\delta$ is the Dirac delta function.

\section{PROPOSED METHOD}

The proposed method can be demonstrated by the following diagram shown in Fig.4. The Brain MR Image Segmentation model using contourlet transform combines variational level set and the contourlet transform. In the first step contourlet transform is applied to the given MR image and the Pyramidal Directional Filter Bank (PDFB), which decomposes images into directional sub-bands at multiple scales. The Contourlet transform divides the original image into four sub-divisions. The approximation subband preserves the information of the original image, and the subbands capture the intensity variations in all the directions.

After CT is applied on the image, contourlet coefficients from the detail sub-bands of all the decomposition levels are used to formulate the energy coefficients. The Standard Deviation $\left(\sigma_{k}\right)$ and/or energy $\left(E_{k}\right)$ of the CT decomposed image on each directional sub-band can be calculated by using,

$$
\begin{gathered}
\sigma_{k}=\sqrt{\frac{1}{N \times N} \sum_{i=1}^{N} \sum_{j=1}^{N}\left(W_{k}(i, j)-\mu_{k}\right)^{2}} \\
E_{k}=\frac{1}{N \times N} \sum_{i=1}^{N} \sum_{j=1}^{N}\left|W_{k}(i, j)\right|^{2}
\end{gathered}
$$

where, $W_{k}$ is the coefficients of $K^{\text {th }}$ contourlet decomposed subband, $\mu_{k}$ is the mean value of $K^{\text {th }}$ sub-band and $N \times N$ is the contourlet decomposed sub-band. The contourlet energy of each pixel is computed by taking the total of the average values in all directions. Each of the contourlet energy value represents the respective pixel's intensity variation information. The final contourlet energies of all the pixels in the image produce a contourlet energy map that shows the various intensity variations present in the image.

In the second stage contourlet energy computed from the first phase will be used to infer the statistical information of the pixels in the image. These contourlet energy values are then formulated into the region based level set method. Assume that an original image $f(x, y)$ contains two structures i.e. desired object and the background. The proposed algorithm segments object from the background with the contour $C$ separating these two structures.

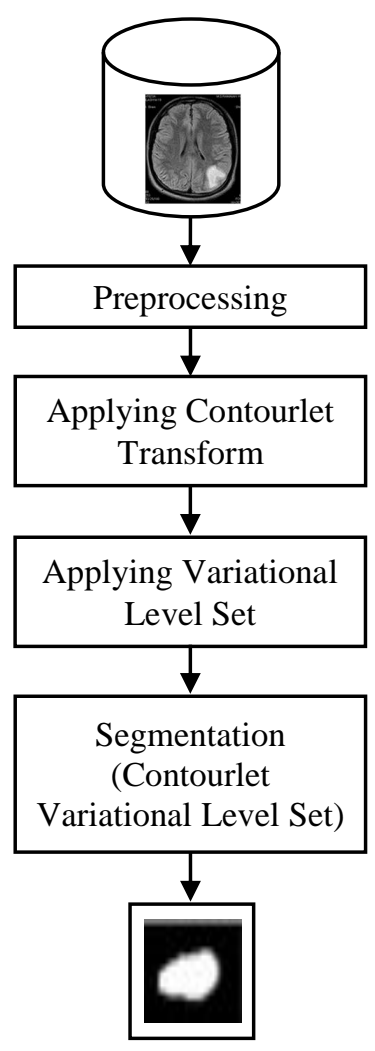

Fig.4. Proposed model for the Brain MR Image Segmentation using Contourlet transform

\section{RESULTS AND DISCUSSION}

This section, presents the experimental results from our proposed segmentation model on brain MR images. In this technique a new region-based active contour model in a variational level set formulation is adapted along with the contourlet transform. In order to demonstrate the strengths of the proposed algorithm based on contourlet transform, several experiments are performed on the MR images of the brain. The proposed algorithm is implemented using Matlab. This proposed model carryout experiments on brain MR images to demonstrate the advantages of the proposed method in solving the sensitivity of the curve initialization problem over the classical speed function and demonstrate the effect of the multiplicative factor.

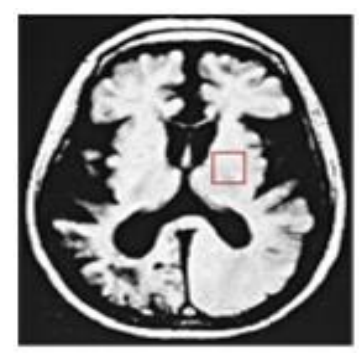

(a) Original image 


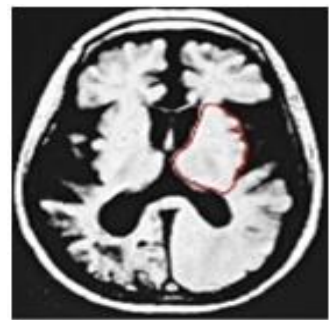

(b) Contour initialization

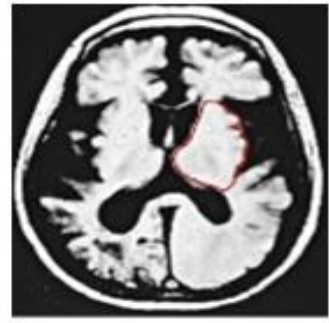

(c) Extracted tumor area

Fig.5. Segmentation results of MR Image 1

The Fig.5 demonstrates the segmentation results of the proposed method for the MR Image 1. The Fig.5(a) is the original MR image, Fig.5(b) indicates the contour initialization and Fig.5(c) shows the final segmentation of the tumor region by the proposed model. Similarly Fig.6 demonstrates the experimental results of MR Image 2.

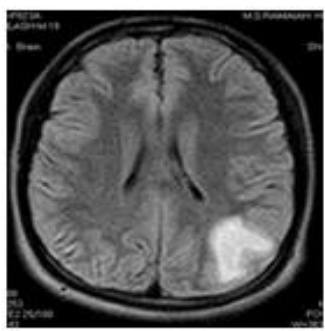

(a) Original image

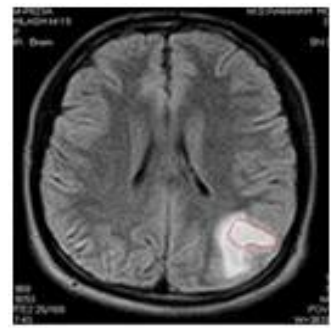

(b) Contour initialization

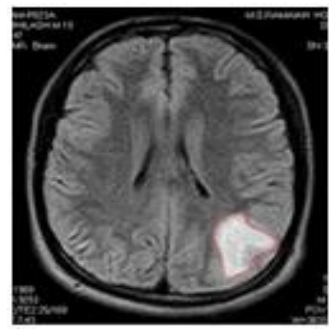

(c) Extracted tumor area

Fig.6. Segmentation results of MR Image 2
The visual results of the proposed method in Fig.6 shows that the proximity of the contour towards the object boundary is more accurate. The reason is that, the proposed method uses variational level sets in conjunction with the contourlet transform. Variational Level set method has many advantages than the Active contours and other edge based methods [23]-[26]. Using this algorithm, smooth contours are well captured and the convergence of the contour is accurate.

Further, the contourlet is applied to the deformable models such as GAC, GVF and Wavelets with Level sets and the performances of these methods are compared with the proposed method for MR image 1 (Fig.7) and 2 (Fig.8) respectively. The experimental results prove that, the sensitivity and segmentation accuracy of the proposed system is improved compare to the existing models. The computational complexity of the proposed method is compared with the various segmentation models available in the literature. The computation time required by the proposed algorithm is very low compared to the modified variational level set proposed by Jayadevappa et al. [26].

The performance measures comparisons of the proposed method are illustrated in Fig.7 for MR Image 1 and 2 respectively.

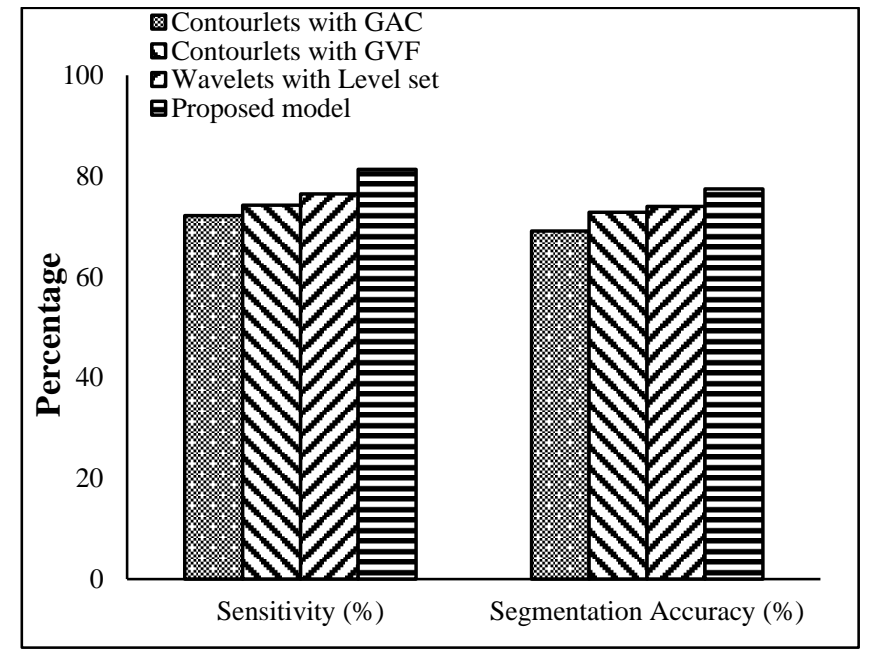

Fig.7. Performance measures comparisons of the proposed method for the MR Image 1

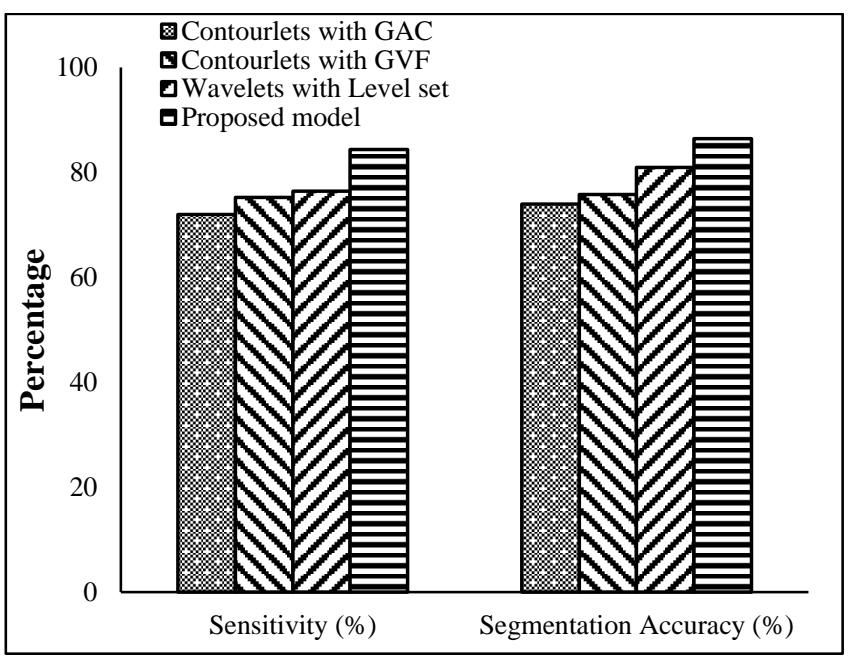

Fig.8. Performance measures comparisons of the proposed method for the MR Image 2 


\section{CONCLUSION}

This paper presented a new variational level set model combined with contourlet transform for brain MR image segmentation. The proposed model is different from other traditional models in two ways. Firstly, before applying active contour model for segmentation of the brain tumor, contourlet transform is used to enhance the weak edges of the images. Secondly, we propose a region fitting term and a global region fitting term adaptively utilizes the intensity information in global and local regions to control the contour evolution. The experimental result indicates that, the proposed model is its ability to extract object boundaries with low-contrast and elongated structures of brain MR images.

\section{REFERENCES}

[1] N.R. Pal and S.K. Pal, "A Review on Image Segmentation Techniques", Pattern Recognition, Vol. 26, No. 9, pp. 1277 1294, 1993.

[2] A.D. Lanterman, U. Grenander and M.I. Miller, "Bayesian Segmentation via Asymptotic Partition Functions", IEEE Transactions on Pattern Analysis and Machine Intelligence, Vol. 22, No. 4, pp. 337-347, 2000.

[3] J.C. Russ, "The Image Processing Handbook", $2^{\text {nd }}$ Edition, CRC Press, 1995.

[4] D.L. Pham, C. Xu and J.L. Prince, "Current methods in Medical Image Segmentation", Annual Review of Biomedical Engineering, Vol. 2, pp. 315-337, 2000.

[5] L.R. Clarke, R. Velthuizen, M. Camacho, J. Heine,M. Vaydianathan, R. Thatcher and M. Silbiger, "MRI Segmentation: Methods and Applications", Magnetic Resonance Imaging, Vol. 13, No. 3, pp. 343-368, 1995.

[6] Daniel J. Withey and Z.J. Koles, "A Review of Medical Image Segmentation: Methods and Available Software", International Journal of Bioelectromagnetism, Vol. 10, No. 3, pp. 125-148, 2008.

[7] O. Gloger, M. Ehrhardt, T. Dietrich, O. Hellwich, K. Graf and E. Nagel, "A Three Stepped Coordinated Level Set Segmentation Method for Identifying Atherosclerotic Plaques on MR Images", Communications in Numerical Methods in Engineering, Vol. 25, No. 6, pp. 615-638, 2009.

[8] V. Pedoia and E. Binaghi, "Automatic MRI 2D Brain Segmentation using Graph Searching Technique", International Journal for Numerical Methods in Biomedical Engineering, Vol. 29, No. 9, pp. 887-904, 2012.

[9] K. Tsai, J. Ma, D. Ye and J. Wu, "Curvelet Processing of MRI for Local Image Enhancement", International Journal for Numerical Methods in Biomedical Engineering, Vol. 28, No. 6-7, pp. 661-677, 2012.

[10] S.O. Dumoulin, R.D. Hoge, C.L. Baker, F.R. Hess, R.L. Achtman and A.C. Evans, "Automatic Volumetric Segmentation of Human Visual Retinotopic Cortex", NeuroImage, Vol. 18, No. 3, pp. 576-587, 2003.

[11] S. Hu and D.L. Collins, "Joint Level Set Shape Modeling and Appearance Modeling for Brain Segmentation", NeuroImage, Vol. 36, pp. 672-683, 2007.

[12] C. Li, C. Xu, C. Gui and M. Fox, "Level Set, Evolution without Re-Initialization: A New Variational Formulation”,
Proceedings of IEEE Computer Society Conference on Computer Vision and Pattern Recognition, pp. 21-26, 2005.

[13] S. Ping and S. Li, "An Improved Mumford-Shah Model and Its Applications to Image Processing with the Piecewise Constant Level Set method", Acta Automatica Sinica, Vol. 33, No. 12, pp. 1259-1262, 2007.

[14] G. Mallat, "A Theory for Multiresolution Signal Decomposition: the Wavelet Representation", IEEE Transactions on Pattern Analysis and Machine Intelligence, Vol. 11, No. 7, pp. 674-693, 1989.

[15] Z. Shi and R. Shibasaki, "An approach to Image Segmentation using Multiresolution Analysis of Wavelets", Proceedings of IEEE International Conference on System, Man, and Cybernetics, pp. 810-815, 1999.

[16] E.J. Candes and D.L. Donoho, "Curvelets a Surprisingly Effective Non-Adaptive Representation for Objects with Edges", PhD Dissertation, Department of Statistics, Stanford University, 1999.

[17] A.B. Watson, "The Cortex Transform: Rapid Computation of simulated Neural Images", Computer Vision Graphics and Image Processing, Vol. 39, No. 3, pp. 311-327, 1987.

[18] F.G. Meyer and R.R. Coifman, "Brushlets: A Tool for Directional Image Analysis and Image Compression", Journal of Application Computation Human Analysis, Vol. 4, No. 2, pp. 147-187, 1997.

[19] M.N. Do and M. Vetterli, "The Contourlet Transform: An Efficient Directional Multiresolution Image Representation", IEEE Transactions on Image Processing, Vol. 14, No. 12, pp. 2091-2106, 2005.

[20] Chencheng Huang and Li Zeng, "An Active Contour Model for the Segmentation of Images with Intensity Inhomogeneities and Bias Field Estimation", PLOS One, Vol. 10, No. 4, pp. 1-6, 2015.

[21] S. Zhou, J. Wang, S. Zhang, Y. Liang and Y. Gong, "Active Contour Model based on Local \& Global Intensity Information for Medical Image Segmentation", Neurocomputing, Vol. 186, pp. 107-118, 2016.

[22] Qiang Zheng, Enqing Dong, Zhulou Cao,Wenyan Sun and Zhenguo Li, "Active Contour Model Driven by Linear Speed Function for Local Segmentation with Robust Initialization and Applications in MR Brain Images", Signal Processing, Vol. 97, pp. 117-133, 2014.

[23] Qi Ge, Chuansong Li, Wenze Shao and Haibo Li, “A Hybrid Active Contour Model with Structured Feature for Image Segmentation”, Signal Processing, No. 108, pp. 147-158. 2015.

[24] Qiang Li, Tingquan Deng, Wei Xie, "Active Contours Driven by Divergence of Gradient Vector Flow", Signal Processing, Vol. 120, pp. 185-199, 2016.

[25] Darya Chyzhyk, Rosalia Dacosta-Aguayo, Maria Mataro and Manuel Grana, "An Active Learning Approach for Stroke Lesion Segmentation on Multimodal MRI Data", Neurocomputing, Vol. 150, pp. 26-36, 2015.

[26] D. Jayadevappa, S. Srinivas Kumar and D.S. Murty, “A Hybrid Segmentation Model based on Watershed and Gradient Vector Flow for the Detection of Brain Tumor", International Journal of Signal Processing, Image Processing and Pattern Recognition, Vol. 2, No. 3, pp. 2942, 2009. 